\title{
Research on the Establishment and Analysis of Small Business Networks
}

\author{
Guozhen Sang \\ ${ }^{1}$ School of Network Security and Informatization, Weinan Normal University, Weinan, 714099 \\ ${ }^{2}$ Engineering Technology Center of Network Security and Informatization, Weinan, 714099
}

Keywords: LAN; Router; Switch; VLAN Technology;

\begin{abstract}
The design builds the enterprise LAN by dividing the IP address and VLAN, and the main configuration of the switch and router. With the establishment of the enterprise network as the center, enterprises can transmit information to each system in a timely and accurate manner, and realize the sharing of resources among various departments. The results show that it can provide theoretical basis and practical guidance for the construction of enterprise network.
\end{abstract}

\section{Introduction}

Due to the weak information base, insufficient funds and lack of technical personnel, enterprises cannot connect their own business and information systems well. The wide application of network can effectively realize resource sharing, technical exchange and information release between work groups, thus organizing production [1]. At the same time, through the network, it is also possible to connect with the outside world to facilitate information exchange between enterprises and outside enterprises.

\section{Corporate LAN Design Goals}

A company is a small business with about 60 employees. The company mainly focuses on sales. The main departments of the company are: Finance Department, Administrative Personnel Department, Logistics Purchasing Department, Supply Department, Production Department, and Marketing Department. With the expansion of the company's scale, the communication technology requirements between the office departments need to be improved [2]. Need to build a complete corporate office network environment.

Through the communication with the company's technical staff, according to the company's requirements, the following design standards are obtained:

(1) To achieve secure access to the Internet, to publish relevant information of the enterprise, and to publicize the culture of the enterprise; to enable normal communication between the enterprise and the user, and to facilitate the user to consult the enterprise. Use the server to store various documents of the enterprise [3].

(2) Due to the rapid development of the company, the company has certain scalability in view of the mission requirements of the enterprise.

(3) Reasonable application and distribution of various resources of the enterprise to achieve effective application of server broadband.

\section{Enterprise LAN Design Requirements}

Configuration: The switches, routers, clients, and servers used should be easy to configure and easy for technical personnel to manage. Device support: device support should be extensive, compatibility between devices should be strong, and the selected operating system and hardware can be highly coordinated. Management: Each device and operating system must be highly managed, and hardware devices can be managed through software. It is convenient for network administrators to manage the entire network system; stability: The designed network should have high stability and reliability to ensure efficient operation of systems and equipment [4]. 


\section{Planning and Design of Enterprise Local Area Network}

\subsection{Enterprise Local Area Network Topology}

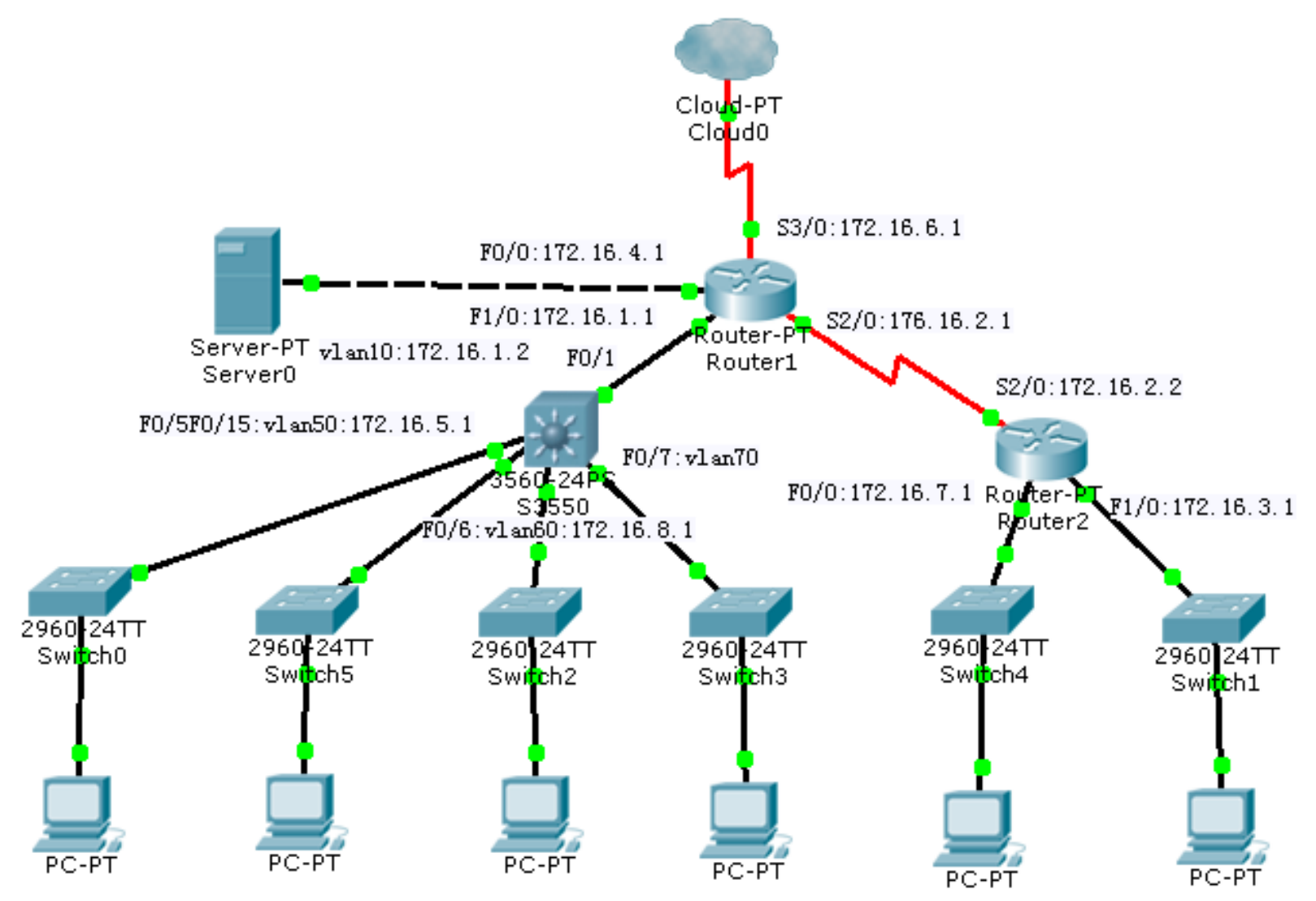

Figure 1 Network topology

\subsection{IP Address and VLAN Division.}

According to the specific situation, the corresponding design scheme [5] is integrated, and the IP addresses are set for each department as shown in the following table:

Table 1 IP address table of each department

\begin{tabular}{|l|l|l|}
\hline department & IP address & Gateway \\
\hline Service department & 172.16 .5 .11 & 172.16 .5 .1 \\
\hline Administrative & 172.16 .15 .11 & 172.16 .15 .1 \\
\hline Logistics Purchasing & 172.16 .8 .11 & 172.16 .8 .1 \\
\hline Supply Department & 172.16 .9 .11 & 172.16 .9 .1 \\
\hline Production department & 172.16 .7 .11 & 172.16 .7 .1 \\
\hline Sales and Marketing & 172.16 .3 .22 & 172.16 .3 .1 \\
\hline
\end{tabular}

According to the mission requirements of the enterprise, the VLANs are divided as follows:

Table 2 VLAN and department list

\begin{tabular}{|l|l|l|l|}
\hline VLAN & port & department & IP of Vlan \\
\hline \multirow{2}{*}{ Vlan 50} & F0/5 & Finance department & 172.16 .5 .1 \\
\cline { 2 - 4 } & F0/15 & politician department & \\
\hline Vlan 60 & F0/6 & Logistics procurement & 172.16 .8 .1 \\
\hline Vlan 70 & F0/7 & Supply Department & \\
\hline$\ldots \ldots$ & $\ldots \ldots$ & $\ldots \ldots$ & $\ldots .$. \\
\hline
\end{tabular}

Mission requirements: The current enterprise requires that the Finance Department and the Administrative Personnel Department be divided into one working system to facilitate resource sharing between the two enterprises, and the Logistics Purchasing Department and the Supply 
Department are not allowed to access this working system. Now configure the switch to achieve this goal.

Create VLAN 50 and divide the ports f0/5 and f0/15 into VLAN 50 to implement communication between the same VLANs [6]. The main steps of the configuration are as follows:

S3550>enable

S3550\#configure terminal

Enter configuration commands, one per line. End with CNTL/Z.

S3550(config)\#vlan 50 //Create vlan 50

S3550(config-vlan)\#exit

S3550(config)\#interface f0/5 //Distribute the f0/5 port to vlan 50

S3550(config-if)\#switchport access vlan 50

S3550(config-if)\#exit

S3550(config)\#

S3550(config)\#int f0/15 //Distribute the f0/15 port to vlan 50

S3550(config-if)\#sw access vlan 50

S3550(config-if)\#exit

S3550(config)\#

The Finance Department (PC1) and the Administrative Personnel Department (PC5) are in the same VLAN 50 and can communicate with each other.

Mission requirements: The finance department and supply department of the two main departments of the design enterprise. For the sake of data security, the finance department and the supply department need to be isolated from each other. Now the switch is configured accordingly to achieve this goal.

Create VLAN 70 and assign the port f0/7 to VLAN 70 to check the communication between different VLANs. The Finance Department (PC1) Supply Department (PC4) is in VLAN 50 and VLAN 70, respectively, and cannot communicate between different VLANs [7].

In a switched network, a physical network is logically divided by VLANs. Different VLANs cannot be directly accessed. You must connect through Layer 3 routing devices. Generally, routers or Layer 3 switches are used to implement mutual access between different VLANs.

A Layer 3 switch can directly access different VLANs by using direct routes. The Layer 3 switch and router have the network layer function, which can perform routing and forwarding according to the IP header information of the data, thereby achieving access between different network segments.

The Layer 3 switch configures the IP address of the interface and uses SVI (switched virtual interface) to implement inter-VLAN interconnection. SVI refers to creating a virtual interface for a VLAN in a switch and configuring an IP address.

Mission requirements: Due to business needs, the current enterprise requires the Finance Department and the Logistics Purchasing Department to communicate with each other. Although the two departments are in different VLANs, data interaction is required. Now configure the switch to achieve this goal. Create VLAN 60, assign the port f0/6 to VLAN 60, and assign IP addresses to VLAN 50 and VLAN 60. View the communication between different VLANs. The Finance Department (PC1) Logistics Purchasing Department (PC3) assigns IP addresses to VLANs 50 and 60 in VLANs 50 and 60, respectively, to enable communication between different VLANs.

\section{Router Configuration}

\subsection{RIP Routing Protocol.}

Routing Information Protocol (RIP) is a distance vector routing protocol used by thousands of networks around the world. The router collects all the different paths to the destination and saves the path information about the minimum number of stations arriving at each destination. Any other information except the best path to the destination is discarded. At the same time, the router also notifies the neighboring routers of the collected routing information by using the RIP protocol. In this way, the correct routing information gradually spread to the entire network. 
RIP currently has two versions [8]: RIPv1 and RIPv2. Compared with RIPv1, RIPv2 has many advantages, so RIPv2 is usually used as long as the device supports it. The biggest difference between RIPv1 and RIPv2 is that RIPv2 supports classless routing because it includes a subnet mask in the routing update, and RIPv1 does not send the subnet mask in the update, so it must rely on the classification default subnet. Mask.

RIPv1 belongs to a classful route and does not support VLSM (variable length subnet mask). RIPv1 updates the routing information in the form of broadcast; the update period is 30 seconds.

RIPv2 is a classless routing protocol that supports VLSM (variable length subnet mask). RIPv2 updates routing information in the form of multicast. RIPv2 also supports port-based authentication to improve network security.

\subsection{Basic Configuration of Enterprise Routers.}

Switch>enable

Switch\#conf t

Enter configuration commands, one per line. End with CNTL/Z.

Switch(config)\#hostname s3550

S3550(config)\#vlan 10

S3550(config-vlan)\#exit

S3550(config)\#vlan 50

S3550(config-vlan)\#exit

S3550(config)\#int f0/1

S3550(config-if)\#sw access vlan 10

S3550(config-if)\#exit

S3550(config)\#int f0/5

S3550(config-if)\#sw access vlan 50

S3550(config-if)\#exit

S3550(config)\#int vlan 10 //Create a vlan virtual interface and configure IP

S3550(config-if)\#

\%LINK-5-CHANGED: Interface Vlan10, changed state to up

S3550(config-if)\#ip address 172.16.1.2 255.255.255.0

S3550(config-if)\#no shutdown

S3550(config-if)\#exit

S3550(config)\#int vlan 50 //Create a vlan virtual interface and configure IP

S3550(config-if)\#

\%LINK-5-CHANGED: Interface Vlan50, changed state to up

S3550(config-if)\#ip address 172.16.5.1 255.255.255.0

S3550(config-if)\#no shutdown

S3550(config-if)\#exit

\section{Equipment Selection and Internet Access}

\subsection{Equipment Selection.}

According to the size of the enterprise and the funding problem, a targeted equipment selection scheme can be developed [9]. The selection method is more flexible. According to the design of the network topology design in this design, the device is selected as follows:

\subsection{Ways to Access the Internet.}

The PSTN access mode is the telephone dial-up access method, which is the commonly used dial-up connection method through MODEM. The required equipment is simple and requires only a modem and a telephone line. Connect the host to the modem and connect the phone line to the modem. This method is the most widely used. The host communicates with the modem and the telephone line to enable Internet access. 
Asymmetric Digital Subscriber Line (Asymmetric Digital Subscriber Line) is referred to as ADSL. This is a new type of data transmission. This approach is named for its asymmetrical upstream and downstream bandwidth. The technology uses frequency division multiplexing to divide the telephone line into three separate parts: uplink, downlink, and telephone. That is, you can make calls while you are on the Internet. Answering calls does not affect the Internet. By separating these three channels, mutual interference between them is avoided. Typically, ADSL provides a maximum upstream rate of $1 \mathrm{Mbps}$ and a maximum downstream rate of $8 \mathrm{Mbps}$. With the latest ADSL2+ technology, the highest downlink rate can be reached at 24Mbps. This laid the foundation for the widespread adoption of this approach.

Integrated Services Digital Network (ISDN), commonly known as "One Line", has a wider range of access than ADSL and LAN access. This method provides high-speed Internet access for those who do not have broadband access, and the speed can reach $128 \mathrm{kbps}$, which is much faster than dialing. But the ISDN method is the same as the phone, and it is charged. Compared with the ADSL method, if the user's monthly online time is less than 20 hours, it is more cost-effective to select the ISDN method [10]. However, since the device is not cheap, and the Internet time is long and expensive, it is not suitable for users who need to access the Internet for a long time.

\section{Conclusion}

In recent years, information networks related to local area networks have developed rapidly. Small enterprises can simultaneously integrate functional business systems on the platform; build various basic information databases and professional databases, and complete resource sharing; and build a complete security system. At present, the performance requirements for various aspects of the security of the local area network have been unprecedentedly increased, and the trend is getting more and more popular. With the gradual expansion of the LAN and the deepening of system applications, the development prospects of LANs will become more and more broad.

\section{References}

[1] Ma Liang. LAN networking technology and maintenance management. Beijing: Publishing House of Electronics Industry, 2009.

[2] Xie Xiren. Computer Network. Beijing: Publishing House of Electronics Industry, 2007.

[3] Zhai Xinyuan, Wu Qiwu, Jiang Lingzhi. Multi-domain optical network secure multicast routing algorithm based on artificial immune and trust. Science Technology and Engineering, 2017, 17(33): 291-296.

[4] Zhang Yanfang. Talking about LAN Technology. Journal of Hebei Tourism Vocational College, 2008, (1): 124-125.

[5] Wang Haozhong, Zhang Yingmei, Wang Wenquan, et al. Research on intelligent substation process layer networking based on multi-link transparent protocol technology. Science Technology and Engineering, 2015, (9): 193-197.

[6] Zhou Wei, Xu Jun. Design and implementation of self-organizing network physical platform based on burst mode. Value Engineering, 2015, (14): 181-182.

[7] Wang Xiaohong. Talking about LAN Technology. Electronic Test, 2014, (s2): 58-60.

[8] Matthew H. Birkner. CISCO Interconnection Network Design. Beijing: Beijing People's Posts and Telecommunications Press, 2000.

[9] Zhai Xinyuan, Wu Qiwu, Jiang Lingzhi. Research and Implementation of ASON Secure Route Simulation Platform Based on NS-2. Optical Communication Research, 2014, (5): 4-10.

[10] Li Qing. Application of VLAN Technology in Enterprise Network. Science and Technology Information, 2010, (16): 195-196. 